\title{
CONTRIBUIÇÕES DAS TECNOLOGIAS DIGITAIS PARA A GESTÃO DO CONHECIMENTO
}

\author{
Guilherme Henrique Müller'1; \\ Vanessa Theis ${ }^{2}$; \\ Dusan Schreiber $^{3}$
}

\begin{abstract}
This paper mains to analyze the digital technologies used in the knowledge management process in the marketing sector of a large higher education institution in southern Brazil. Together, it seeks to identify possible points for improvement in the observed practice and the theoretical proposal developed during the study. Empirical data were collected through semistructured interviews and complemented with documentary surveys and participant observation on the spot. As main results, it is highlighted that a potential space was identified for the training of professionals on the use of web 2.0 tools and collaborative tools, in order to obtain greater benefit from their functionalities.
\end{abstract}

Keywords: Knowledge management; Digital Technologies.

\section{Resumo}

O objetivo deste artigo é analisar as tecnologias digitais utilizadas no processo de gestão do conhecimento no setor de marketing de uma instituição de ensino superior de grande porte do sul do Brasil. Juntamente, busca-se identificar possíveis pontos de melhoria na prática observada e a proposta teórica desenvolvida no decorrer do estudo. Os dados empíricos foram coletados por meio de entrevistas semiestruturadas e complementados com o levantamento documental e observação participante in loco. Como principais resultados, destaca-se que foi identificado um espaço potencial para capacitação dos profissionais sobre a utilização das ferramentas da web 2.0 e ferramentas colaborativas, a fim de obterem maior proveito das funcionalidades das mesmas.

Palavras-chave: Gestão do Conhecimento; Tecnologias Digitais.

\section{INTRODUÇÃO}

As mudanças no mercado global, movido pelos processos de transformação digital, cada vez mais forçam às organizações a se reinventarem. Neste cenário, as agências de publicidade

\footnotetext{
${ }^{1}$ Comunicação Social - Universidade Feevale, Novo Hamburgo - Brasil. E-mail: gmullernh@feevale.br

${ }^{2}$ Indústria Criativa - Universidade Feevale, Novo Hamburgo - Brasil. E-mail: vanessat@feevale.br

${ }^{3}$ Indústria Criativa - Universidade Feevale, Novo Hamburgo - Brasil. E-mail: dusan@feevale.br
} 
e departamentos de marketing encontram um ambiente de negócios complexo, pois suas rotinas são variáveis e precisam ofertar um serviço cujo resultado é, em grande parte, subjetivo. Além disso, Baker et al. (2020) destacam que a popularização da internet, computadores, dispositivos móveis e outras tecnologias, impactou as relações de trabalho, ampliando os requisitos básicos do profission, aumentando a rotatividade dos profissionais, contribuindo para um ambiente com problemas de gestão.

Neste sentido, Intezari, Taskin e Pauleen (2017) destacam que os centralizam o conhecimento, muitas vezes por medo de perderem o poder ou por não perceberem o valor do conhecimento para seus pares. Destarte, em um mercado em que a valorização da eficácia na comunicação é maior do que o potencial criativo por si só, o sucesso de uma organização está diretamente ligado à sua capacidade de criar e transmitir conhecimento dentro da sua estrutura.

Neste sentido, este trabalho tem como objetivo analisar as tecnologias digitais utilizadas no processo de gestão do conhecimento no setor de marketing de uma instituição de ensino superior de grande porte do sul do Brasil. Juntamente, busca-se identificar possíveis pontos de melhoria na prática observada e a proposta teórica desenvolvida no decorrer do estudo. Após esta introdução, esta pesquisa apresenta a seguinte estrutura: a contextualização da Gestão do Conhecimento, bem como uma abordagem sobre as contribuições das tecnologias digitais para a gestão do conhecimento. A seguir, são elencados os procedimentos metodológicos, e, por fim, expõe-se as análises e discussões dos resultados. Após a descrição e apresentação do corpus pesquisado, estão descritas as considerações finais em relação ao objetivo, suas contribuições, e sugestões para investigações futuras.

\section{A GESTÃO DO CONHECIMENTO}

Conforme Probst, Raub e Romhardt (2009), o conhecimento é um recurso econômico que possibilita a criação de novas estratégias competitivas e inovações. Neste sentido, Takeuchi e Nonaka (2009) esclarecem que as organizações aplicam esforços para tratá-lo como recurso gerenciável. Logo, a Gestão do Conhecimento pode ser definida como o processo de criar continuamente novos conhecimentos, disseminando-os na organização e incorporando-os em novos produtos, serviços, tecnologias e sistemas, auxiliando, então, para perpetuar as mudanças desenvolvidas internamente. 
O conhecimento poderá ser representado por meios formais ou informais, e para esse entendimento, determina-se a classificação de conhecimento em explícito e tácito. Para Fialho et al. (2006) o conhecimento explícito diz respeito à racionalidade que envolve o conhecimento de fatos, sendo adquirido principalmente pela informação. Em contrapartida, para Falsarella, Januzzi e Sugahara (2016), o conhecimento tácito representa uma qualidade pessoal, o que lhe torna mais difícil de comunicar. Takeuchi e Nonaka (2009), descrevem o como altamente pessoal, fragilizando a formalização e o compartilhamento.

A aquisição do conhecimento pode resultar da participação individual e da interação com tarefas, rotinas, tecnologias, recursos e pessoas, dentro de um contexto específico, segundo Yazdi e Soltanali (2019). Os indivíduos assimilam os conhecimentos, mas cabe às organizações criar o contexto favorável para que isso ocorra. Assim, o principal papel da gestão é o de estabelecer a coordenação necessária para a integração do conhecimento. Por fim, Ahn et al. (2019), destacam que o processo de transferência não depende apenas de características cognitivas do usuário que embasa a interpretação, mas também da forma de como o mesmo é disponibilizado ao usuário, ou seja, da codificação empregada para representar aquele conhecimento específico.

\subsection{CONTRIBUIÇÕES DAS TECNOLOGIAS DIGITAIS PARA A GESTÃO DO CONHECIMENTO}

Para Hinchcliffe (2013), existe uma grande oportunidade para aumentar os resultados dos times de uma organização com ferramentas que reduzam o tempo e a distância entre os indivíduos, definam caminhos claros para a melhora de performance e tornem a informação de fácil acesso. $\mathrm{O}$ autor recomenda o uso de ferramentas digitais para reduzir os conflitos que podem emergir dos preceitos constituídos dentro da cultura organizacional existente e potencializar a capacidade produtiva da organização. Contudo, Hinchcliffe (2018) complementa que para ocorrer a transformação digital, facultando às empresas constituir uma estrutura voltada às experiências digitais e holísticas, deve acontecer antes uma transformação cultural, de processos e tecnologia no ambiente organizacional.

A popularização dos microcomputadores e da internet, oportunizou a transição da Web 1.0 para Web 2.0, a web como plataforma, ampliou a conectividade entre os usuários, 
estabelecendo espaços de diálogo (Wal, 2007; Cook, 2008b; Motta et al., 2011). Em se tratando de ferramentas e técnicas de tecnologias digitais aplicadas à administração empresarial, Rossini e Palmisano (2013), destacam os Sistemas de Informação. McAfee (2006) propõe o conceito de Enterprise 2.0, compreendendo o uso de ferramentas da Web 2.0 no contexto empresarial. Em complemento, Cook (2008b) apresenta o conceito de SLATES (Search, Links, Authoring, Tags, Extensions, Signals) para identificação das características de ferramentas do contexto da Enterprise 2.0:

- Search: vez que as informações tenham valor dentro da plataforma é necessário que o usuário possa encontrá-las;

- Links: auxiliam a interconectar a estrutura das informações;

- Authoring: permite a criação de conteúdo por parte dos usuários;

- Tags: a categorização dos conteúdos deve ser através de tags, baseado em Folksonomia. Ao contrário da taxonomia clássica, que é criada por um grupo de gestores e permanece engessada, a folksonomia é desenvolvida pelos usuários ao longo do tempo de uso e potencializa a construção social do conhecimento e proporciona mecanismos eficazes para a circulação e recuperação de informações (Meira et al., 2011);

- Extensions: podem ser estendidas através de novas funcionalidades pelos usuários;

- Signals: informar ao usuário quando novos conteúdos foram inseridos na plataforma.

Com relação às redes internas, que permitem a conexão entre os colaboradores da empresa, Hinchcliffe (2014), Mäntymäki, Riemer (2016) e Azaizah et al. (2018) destacam que as Enterprise Social Networks (ESNs) possuem dinâmicas similares às redes sociais tradicionais ao permitir a comunicação, compartilhamento e colaboração entre os indivíduos e apresentam um potencial de melhora na produtividade, pois auxiliam no fluxo e intercâmbio do conhecimento (Von Krogh, 2012; Wong et al., 2015). Wal (2018) corrobora afirmando que existem diversas plataformas baseadas nos modelos de interação, com destaque para o modelo cooperativo social fundamentado no conceito de um ou mais tópicos, sobre os quais os indivíduos discutem e compartilham, auxiliando no entendimento e coesão dos indivíduos.

Fuks et. al. (2011) descrevem o modelo 3C de colaboração, proposto por Ellis et. al. (1991) composto por três pilares de suporte ao trabalho em grupo. O modelo 3C compreende a Comunicação, através da negociação, comprometimento e diálogo entre os agentes envolvidos; 
a Coordenação, ao colocar as pessoas, tarefas e recursos em ordem para atingir os objetivos propostos; e a Cooperação, a atuação em conjunto em um espaço compartilhado. Cook (2008a) estende o modelo de colaboração $3 \mathrm{C}$ para $4 \mathrm{C}$, fragmentando a Cooperação em Cooperação e Colaboração em itens distintos, onde a cooperação ocorre de forma individual e a colaboração, de forma coletiva. Ao tratar de softwares colaborativos, Cook (2008a) classifica-o como um software social com base em quatro principais funções: comunicação, colaboração, cooperação e conexão.

Para apoiar o processo de construção do conhecimento coletivo, Borges (2011) recomenda o uso de wikis, como forma de armazenar e validar o conhecimento distribuído entre os indivíduos. De Sordi (2015), Moffett, McAdam e Parkinson (2004) Polizelli e Ozaki (2008) e Pereira e Bellini ( 2008) apresenta três grupos para classificação de softwares de apoio à gestão do conhecimento nas organizações:

1. Ferramentas colaborativas: compreendendo os softwares de trabalho em grupo (groupware), sistemas de vídeo conferência e brainstorming, diretórios de conhecimento, intranets e extranets;

2. Ferramentas de gestão de conteúdo: agem através de provedores de informação, agentes e filtros, sistemas de gestão de conteúdo, sistemas de automação de escritório e sistemas de publicação eletrônica.

3. Sistemas de inteligência de negócio: com data warehouses $^{4}$, data mining, sistemas de suporte à decisão, sistemas baseados em conhecimento e ferramentas de workflow.

Cumpre destacar que o avanço das tecnologias digitais permitiu a utilização de tecnologias como mediadoras das Comunidades de Prática (COPs), criando assim, as Comunidades de Prática Virtuais (COPVs). Estas unem indivíduos da mesma forma que as comunidades de prática tradicionais, contudo, apresentam a mediação por tecnologias e permitem a interação de forma assíncrona ( Wenger; Mcdermott; Snyder, 2002). A utilização dessas comunidades faculta a criação de repositórios de conhecimento dentro da organização, contribuindo para as práticas de gestão do conhecimento.

${ }^{4}$ Depósitos de dados que objetivam armazenar informações e dados sobre o negócio da organização, permitindo a extração de relatórios e históricos de forma estruturada. 


\section{MÉTODO}

Com o propósito de adotar as decisões adequadas no processo de investigação científica, a fim de que aos resultados possa ser conferido o rigor científico, os autores optaram pela realização do estudo de caso único e abordagem qualitativa e quantitativa. Em atendimento à recomendação de autores que versam sobre o método de estudo de caso, com destaque ao Yin (2015), os pesquisadores realizaram a triangulação de dados, por meio de entrevista em profundidade, levantamento documental e observação participante.

O universo adotado para a realização desta pesquisa foi o setor de marketing que adota uma metodologia de trabalho híbrida entre uma agência de publicidade e um modelo de gestão de marketing orientado à comunicação integrada. O setor apresenta um quadro funcional de 36 colaboradores, sendo que 12 foram entrevistados, entre os meses de setembro e outubro de 2019. O setor apresenta uma divisão baseada em núcleos sob a supervisão de uma coordenação, contando com um núcleo de atendimento e criação, além de núcleos específicos para atender demais demandas relacionadas aos serviços de um departamento de marketing com base em parte dos componentes da comunicação integrada, com os núcleos de digital, produtora audiovisual, relações públicas e endomarketing. O setor conta também com um núcleo voltado à assessoria de imprensa. Os núcleos que constituem o setor são divididos em funções complementares, a fim de contribuir ao todo do processo de marketing da organização, que é uma Instituição de Ensino Superior, localizada em Novo Hamburgo.

A coleta de dados iniciou através da pesquisa documental no documento intitulado "Manual do Marketing”, que fornece a definição das atribuições e atividades do setor dentro da instituição. Após a análise inicial do documento, buscou-se compreender dentro dos processos realizados pelos núcleos, assim como, processos emergentes e/ou não documentados, a natureza dos conhecimentos envolvidos, a incidência de práticas de gestão do conhecimento e o uso de ferramentas tecnológicas digitais.

Com base no embasamento teórico, foi possível estabelecer uma tabela com as categorias de análise para a realização de entrevistas semiestruturadas com os núcleos, com a finalidade de avaliar se existem determinadas práticas de gestão do conhecimento pela perspectiva dos entrevistados. Assim, foi estabelecida uma relação ternária de intensidade de adesão a determinado indicador, variando entre "sim" ( 1 ponto), 'relativamente" ( 0,5 pontos) e 
"não" (0 pontos), objetivando a compreensão da incidência dos fatores perante o número total de processos mapeados pelo manual e durante as entrevistas, conforme demosntra o Quadro 1. As entrevistas foram realizadas com dois ou mais entrevistados por núcleo, a fim de incentivar o diálogo e reflexão entre os participantes.

Quadro 1 - Roteiro da entrevista

\begin{tabular}{|c|c|}
\hline $\begin{array}{l}\text { Natureza do conhecimento: } \\
\text { Takeuchi e Nonaka (2009), Fialho et al. (2006), } \\
\text { Falsarella, Januzzi e Sugahara (2016) }\end{array}$ & Tácito, explícito, organizacional e individual \\
\hline $\begin{array}{l}\text { Importância Estratégica de determinado conhecimento: } \\
\text { Probst, Raub e Romhardt (2009) Fialho et al. (2006) }\end{array}$ & Criação de valor; transferibilidade \\
\hline $\begin{array}{l}\text { Sobre o processo de Gestão de conhecimento: } \\
\text { Takeuchi e Nonaka (2009), Borges (2011), De Sordi } \\
\text { (2015), Moffett, McAdam e Parkinson (2004), Polizelli e } \\
\text { Ozaki (2008), Pereira e Bellini ( } 2008)\end{array}$ & Documetado, recuperável e difundido \\
\hline $\begin{array}{l}\text { Comunidades de prática: } \\
\text { Wenger, Mcdermott e Snyder (2002) }\end{array}$ & Apresentam modelos de reflexão entre os setores \\
\hline $\begin{array}{l}\text { Ferramentas de TI aplicadas à administração empresarial } \\
\text { e gestão do conhecimento: } \\
\text { Rossini e Palmisano (2013), McAfee (2006), Cook } \\
\text { (2008a;b), Meira et al. (2011), Hinchcliffe (2014), } \\
\text { Mäntymäki, Riemer (2016), Azaizah et al. (2018), } \\
\text { Von Krogh (2012) Wong et al. (2015), Wal (2018) }\end{array}$ & $\begin{array}{l}\text { Sistemas de informação, Ferramentas } \\
\text { colaborativas, redes empresariais, chats, fóruns de } \\
\text { discussão, blogs, worflow, ferramentas de busca, } \\
\text { integração com sistemas corporativos, sistemas de } \\
\text { inteligência de negócio, mapas de conhecimento }\end{array}$ \\
\hline $\begin{array}{l}\text { Características das ferramentas de gestão do } \\
\text { conhecimento: } \\
\text { Fuks et. al. (2011), Ellis et. al. (1991),Cook (2008a) }\end{array}$ & $\begin{array}{l}\text { Oportuniza a colaboração, modelo } 3 \mathrm{C} \text {, Web } 2.0 \text {, } \\
\text { plataformas sociais. }\end{array}$ \\
\hline
\end{tabular}

Fonte: Dados da pesquisa.

Cumpre destacar que cada uma das categorias de análise elencadas no Quadro 1, foram também analisadas à luz de processos descritos no Manual de Marketing da Instituição, contudo, devido ao extenso formato da planiha final, optou-se por sumarizar apenas as categorias e autores utilizados. Para a análise dos dados, através da análise de conteúdo, as respostas foram agrupadas por similaridade da área de conhecimento, visando condensar os resultados em 3 campos com seus respectivos itens: i) Aspectos Gerais sobre o conhecimento: engloba-se a natureza do conhecimento, sua criticidade para a operação e as práticas de gestão do conhecimento; ii) Gestão do conhecimento: os conceitos relativos ao conhecimento, sua natureza e as qualidades de transferibilidade e complexidade; iii) Utilização de Tecnologias Digitais. Para facilitar a compreensão das relações adotadas, desenvolveu-se um diagrama, que pode ser verificado a seguir, que aponta a relação entre os componentes das categorias e seus respectivos autores. 
Figura 1 - Relações em utilização de tecnologias digitais para definição do campo

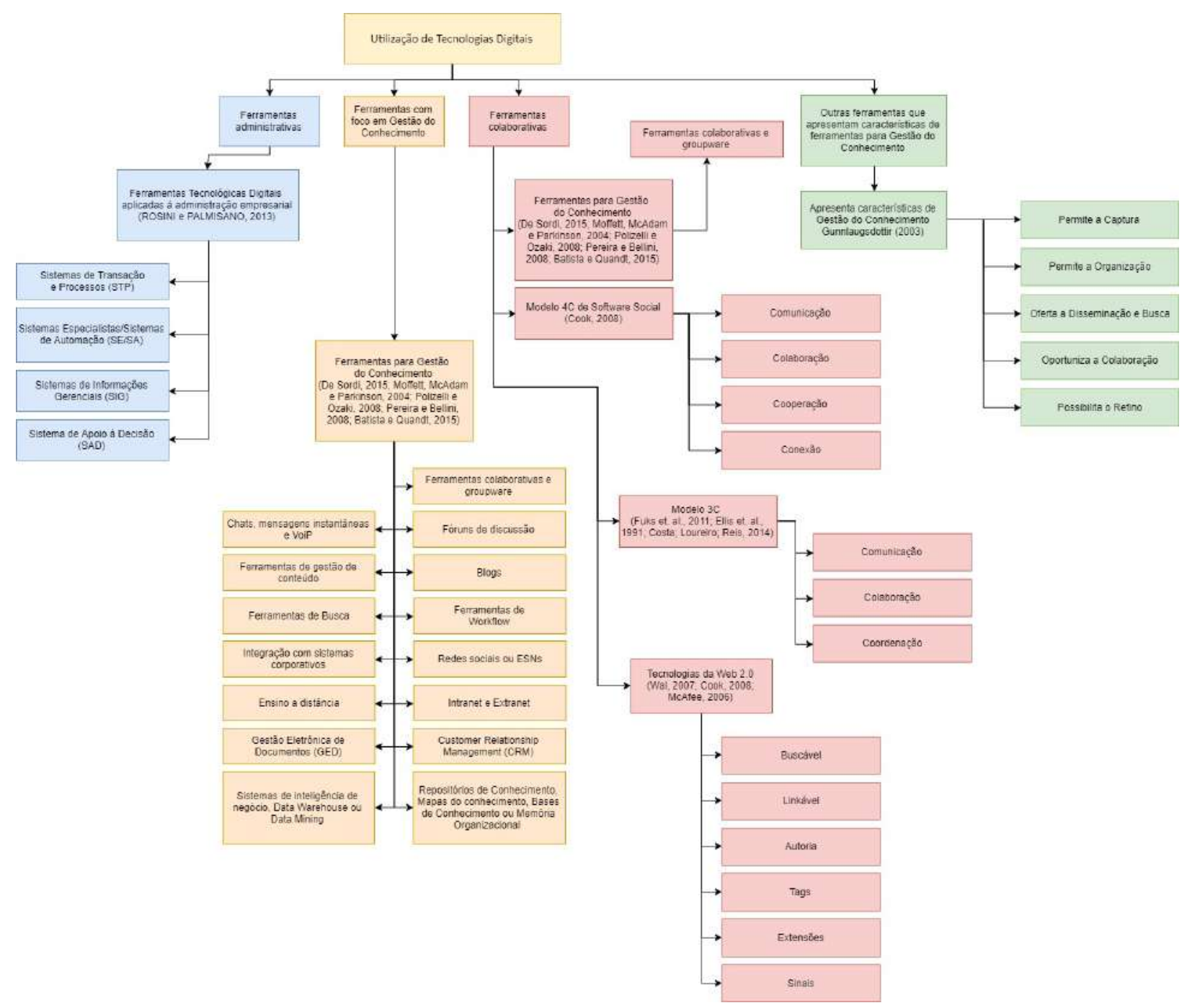

Fonte: Dados da pesquisa.

Para realizar a avaliação das tecnologias utilizadas, devido ao ser caráter técnico, foram questionadas quais as principais ferramentas utilizadas em cada processo e a forma de uso, para então, a partir disso, realizar-se a análise de enquadramento das funcionalidades dentro das 
categorias acima propostas. Desta forma, foi possível identificar a quantidade de processos que apresentam o uso de determinada funcionalidade em sua operação. Para a pontuação das categorias foi considerado o maior valor ou soma dos itens.

\section{ANÁLISE E DISCUSSÃO DOS RESULTADOS}

Os entrevistados relataram a utilização de sistemas administrativos e integrações com sistemas corporativos, apontando para um alto grau de informatização nos processos. $\mathrm{Na}$ dimensão das ferramentas colaborativas, ao se analisar a quantidade de processos que apresentam o uso de ferramentas de caráter colaborativo, não foi possível observar uma relação direta entre quantidade de processos que exibem conhecimento explícito e o uso dessas ferramentas, conforme a figura 2:

Figura 1 - Relação entre processos mapeados, natureza e difusão do conhecimento e o uso de ferramentas colaborativas

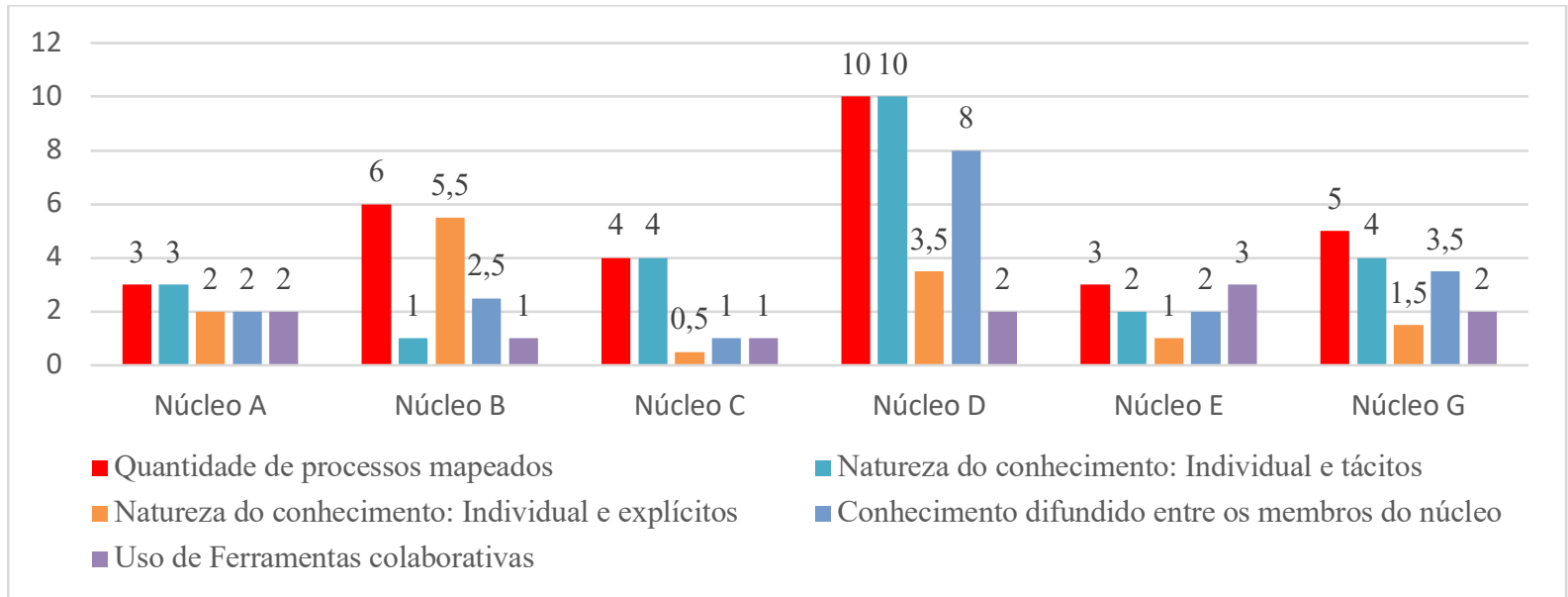

Fonte: Dados da pesquisa.

Tais resultados podem estar atrelados a fatores culturais, comportamentais ou de processos (HINCHCLIFFE, 2013) para maior adoção destas ferramentas, ou fatores culturais relativos à uma visão de não importância para a documentação, ou a não utilização delas para esse fim, ou, ainda, uma possível incompatibilidade dos tipos de conhecimento envolvidos na prática publicitária e o uso das mesmas. Todavia, o uso de ferramentas digitais administrativas, foi identificada em quase todos os processos, demonstrando alto grau de informatização dos processos na instituição. 
Conforme indica a Figura 3, o uso de ferramentas da web 2.0 também aparece em grande parte dos processos, corroborando as bases conceituais de Wal (2007), Cook (2008a) Motta et al. (2011) de que a web enquanto plataforma, ampliou o espaço para diálogos. Dessa forma, a utilização dessas ferramentas possui alto potencial de contribuição para aumentar a taxa de sucesso dos projetos realizados no setor, sendo esse formato um dos mais frequentes realizados pelos profissionais da área de publicidade e marketing. Assim, cabe o incentivo ao uso destas ferramentas e a exploração de suas funcionalidades, que, muitas vezes, não são utilizadas ao seu completo potencial.

Figura 2 - Uso de ferramentas da web 2.0 nos processos

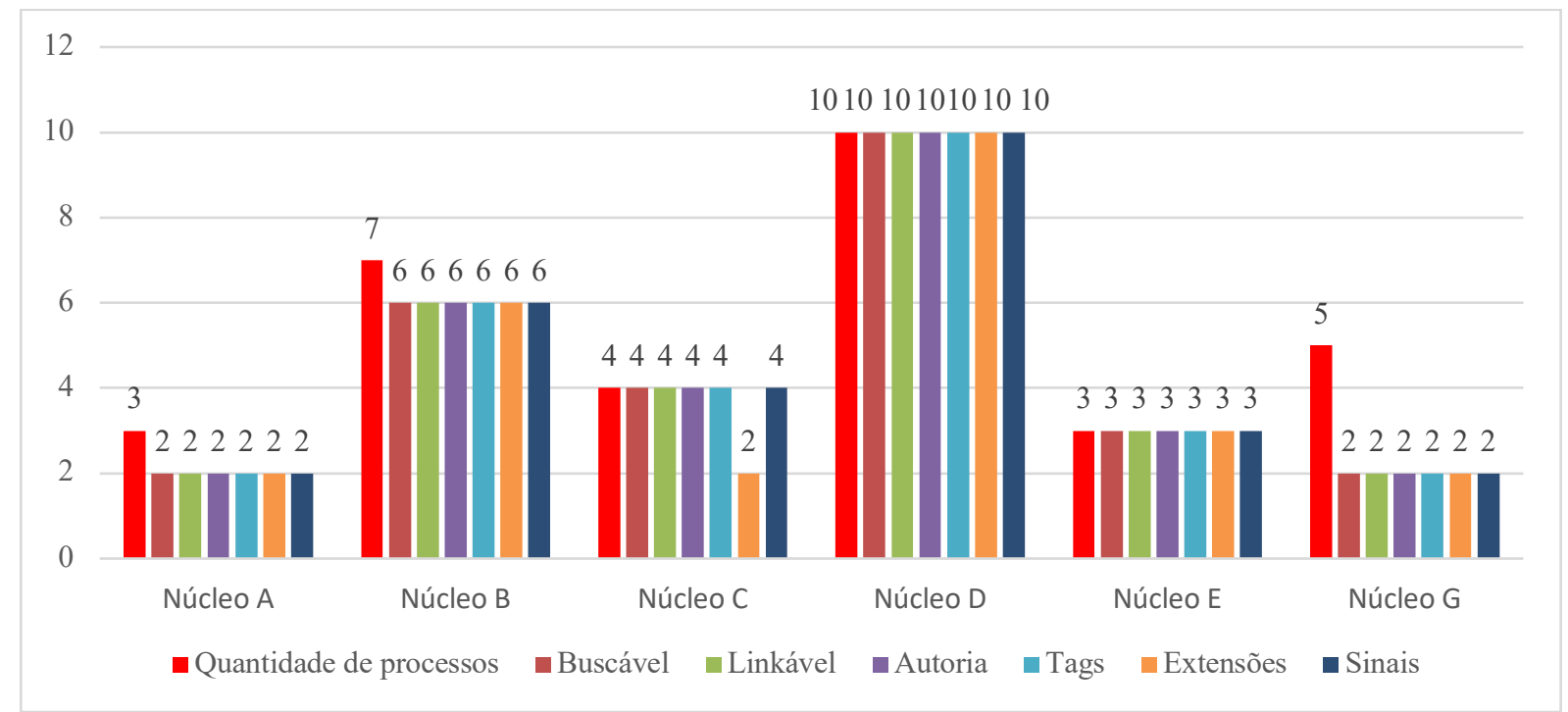

Fonte: Dados da pesquisa.

Dentro dos demais resultados observados, o Quadro 2 demonstra quais ferramentas da web 2.0 são utilizadas, e, com qual propósito.

Quadro 2- Ferramentas web 2.0 utilizadas 


\begin{tabular}{|l|l|}
\hline Tipo de Ferramenta & \\
\hline \multirow{3}{*}{$\begin{array}{l}\text { Chat } \\
\text { comunicação }\end{array}$} & $\begin{array}{l}\text { Apresentam potencial para gestão do conhecimento, uma vez que registram as mensagens } \\
\text { trocadas e permitem sua recuperação através de buscas posteriores. }\end{array}$ \\
\cline { 2 - 3 } Workflow & $\begin{array}{l}\text { Os métodos mais tradicionais de comunicação assíncrona, como o e-mail aparecem com } \\
\text { frequência. }\end{array}$ \\
\hline & $\begin{array}{l}\text { Ferramentas de caráter cooperativo, no formato tradicional: } \\
\text { i) Apresentam modos de trabalho individualizado, onde a cooperação ocorre quando cada } \\
\text { pessoa realiza sua parte da tarefa; } \\
\text { ii) Atuam como suporte às operações, registrando as atividades de trabalho e com atuação } \\
\text { de forma hierárquica. }\end{array}$ \\
\cline { 2 - 2 } & $\begin{array}{l}\text { Ferramentas de caráter colaborativo, permitindo a gestão visual das tarefas, oportunizam } \\
\text { maior flexibilidade na execução das atividades. }\end{array}$ \\
\hline
\end{tabular}

Fonte: Dados da pesquisa.

Cumpre destacar que, a maior parte dos processos é realizada por meio de ferramentas propícias para a gestão do conhecimento, contudo, um baixo índice de processos encontra-se sendo gerido. Assim, observa-se que existe uma oportunidade para a utilização do potencial destas ferramentas. Ressalta-se que em nenhum dos núcleos foi identificado o uso de ESNs ou redes sociais corporativas, demonstrando a possibilidade para adequação ao modelo de Hinchcliffe (2014).

\subsection{REFLEXÕES E RECOMENDAÇÕES}

Com base nas práticas verificas no setor, propõem-se soluções para equilibrar os indicadores observados: alto índice de conhecimentos tácitos e poucos documentados. Ao passo que foi observado alto nível de utilização de ferramentas da web 2.0, foi identificado o potencial para que suas funcionalidades SLATE (Busca, links, autoria, tags, extensões e sinais) sejam melhores exploradas no dia a dia das atividades.

Assim, por meio do uso de ferramentas colaborativas baseadas no modelo 4C (Cook, 2008a), apresenta-se um potencial de difusão e circulação do conhecimento. A escolha dessas ferramentas pode ser oirientada por funcionalidades específicas ou a solução de problemas de caráter mais subjetivo, como sugerido por Hinchcliffe (2013). Com base nos resultados apontados previamente, a escolha pode ser pautada pela solução dos problemas de difusão do conhecimento, construção de bases de conhecimento e memória organizacional e criação de comunidades de prática virtuais. Também, se observa a oportunidade para adaptar os métodos de utilização das ferramentas atuais para compreender a gestão do conhecimento, uma vez que muitas apresentam as funcionalidades, mas não são utilizadas para gerir o conhecimento explícito nem organizacional. 
Neste contexto, é válido destacar que Hinchcliffe $(2013 ; 2018)$ aponta um futuro dos negócios extremamente conectado, baseado na gestão de comunidades internas ou externas. A adoção de um formato voltado à gestão de comunidades apresenta um grande potencial para auxiliar no fluxo e transformação do conhecimento entre os indivíduos, visto que potencializa a criação de novos conhecimentos a partir de fontes externas, amplia as conexões internas na organização, fomentando o surgimento de comunidades de prática. Assim, as ferramentas de groupware e demais ferramentas colaborativas, apresentam grande potencial para suporte à gestão destas comunidades.

Nas redes internas da organização, a utilização de uma enterprise social network (ESN) demonstra grande potencial para a movimentação da espiral do conhecimento entre os indivíduos à nível coletivo e organizacional (Borges, 2011) e como fomento à criação de novas comunidades de prática virtuais ( Wenger; Mcdermott; Snyder, 2002). A escolha de uma ESN para este caso pode ser realizada como sugere Wal (2018), visando a adoção de uma plataforma do modelo cooperativo social, uma vez que tal modelo auxilia no entendimento e coesão dos indivíduos, ou do modelo colaborativo, que objetiva agrupar diferentes ideias e entendimentos em um mesmo lugar.

A implantação do uso de ferramentas de workflow com funcionalidades sociais, apresenta uma oportunidade para implementação de uma cultura $W o L^{5}$ e ampliação dos espaços de discussão, principalmente, no campo virtual. A utilização de aplicativos colaborativos nas operações (socially-enabled $L O B^{6}$ apps $^{7}$ ) representa uma possibilidade para ampliar a quantidade de conexões entre os indivíduos, fomentando a criação de comunidades de prática e facilitando a circulação do conhecimento.

Nas redes híbridas, a criação de comunidades de fornecedores e inovação demonstra potencial para integração e promoção da agilidade nas relações e parcerias com terceiros, facilitando, também, a prospecção e identificação de potenciais parceiros de negócio e

${ }^{5}$ WoL, do inglês, Working out Loud, é metodologia de trabalho colaborativo, onde se busca a confiança e cooperação para obtenção de resultado.

${ }^{6}$ Do inglês, Line of Business, pode ser traduzido como: linha de frente do negócio.

${ }^{7}$ Do inglês, pode ser traduzido como: aplicativos de linha de frente de negócio com funcionalidades sociais. 
fornecedores. Nas redes externas, principalmente, dentro da alçada do departamento, constatouse o desenvolvimento de programas para gestão de influenciadores, dos divulgadores de marca e da comunidade de clientes, bem como a criação de um portfólio de marketing social apresentam grande oportunidade para potencialização dos esforços de marketing. Como programas de suporte, a implantação de times de gestão de comunidades e mídias sociais de excelência e um centro de negócios digitais também oportunizam o desenvolvimento das atividades de marketing na organização.

Para atingir o cenário acima descrito, mostra-se necessário mover o modelo organizacional de uma estrutura de silos para uma estrutura voltada à experiência. Apesar da abordagem voltada para uma visão organizacional, tal lógica também aparenta ser aplicável ao objeto analisado, uma vez que, os núcleos que o compõem podem ser relacionados com a estrutura clássica de silos, logo, torna-se possível adotar a estratégia de transformação das frentes de negócio em experiência. Tal transformação ocorre através dos pilares de experiência do consumidor, experiência do colaborador e experiência do fornecedor ou parceiro, e objetiva a adaptação ao novo cenário competitivo global.

\section{CONSIDERAÇÕES FINAIS}

Através deste estudo, foi identificado um espaço potencial para capacitação dos profissionais da área sobre a utilização das ferramentas da web 2.0 e ferramentas colaborativas, a fim de obterem maior proveito das funcionalidades das mesmas. Com base na natureza da profissão, os profissionais podem se beneficiar da memória organizacional e mapas do conhecimento. As dinâmicas de projeto, adotadas por agências e departamentos de marketing, proporcionam um ambiente propício para a utilização de metodologias de revisão e construção de bases e mapas de conhecimento e outras metodologias apresentadas pelos autores citados neste trabalho.

No campo da análise entre o uso de ferramentas tecnológicas e gestão do conhecimento, entende-se como uma oportunidade para uma metodologia de trabalho mais rigorosa, voltada para uma identificação de componentes das ferramentas, principalmente, as colaborativas, que possam auxiliar nos processos de gestão do conhecimento. Da mesma forma, acredita-se na possibilidade de desenvolvimento de uma metodologia de trabalho que permita a desconstrução 
dos processos nos conhecimentos envolvidos, com isso abrindo possibilidades para a inovação nos mesmos.

No campo da gestão do conhecimento, compreende-se que há espaço para o desenvolvimento de metodologias que auxiliem na transferência de conhecimento de caráter, bem como, o desenvolvimento de ferramentas digitais que permitam a explicitação do conhecimento de forma automatizada com maior riqueza do que uma simples descrição. Também, através dos modelos 3C e 4C, pode-se explorar o desenvolvimento de ferramentas tecnológicas para o apoio ao ensino e difusão do conhecimento entre os profissionais de publicidade e propaganda.

\section{REFERÊNCIAS}

Ahn, S., Hu, S. X., Damianou, A., Lawrence, N. D., \& Dai, Z. (2019). Variational information distillation for knowledge transfer. In Proceedings of the IEEE Conference on Computer Vision and Pattern Recognition (pp. 9163-9171).

Azaizah, N., Reychav, I., Raban, D. R., Simon, T., \& McHaney, R. (2018). Impact of ESN implementation on communication and knowledge-sharing in a multi-national organization. International Journal of Information Management, 43, 284-294

Baker, N. B., Said Boustany, M., Khater, M., \& Haddad, C. (2020). Measuring the indirect effect of the Internet on the relationship between human capital and labor productivity. International Review of Applied Economics, 1-18.

Bellini, C. G. P., \& Pereira, R. De C. De F. (2008). As redes como tecnologia de apoio à gestão do conhecimento. In: Organizações do Conhecimento: infra-estrutura, pessoas e tecnologia. São Paulo: Saraiva.

Cook, N. (2008a). Social Software in the Enterprise. Enterprise 2.0. How Social Software Will Change the Future of Work, p. 33-40.

Cook, N. (2008b). Enterprise 2.0: How social software will change the future of work. Gower Publishing, Ltd..

De Sordi, J. O. (2015). Administração da informação : fundamentos e práticas para uma nova gestão do conhecimento, 2015. Saraiva Educação SA.

Ellis, C. A., Gibbs, S. J., \& Rein, G. (1991). Groupware: some issues and experiences. Communications of the ACM, 34(1), 39-58.

Falsarella, O. M., \& Roberta, C. (2016). Gestão do conhecimento: um estudo de modelos e sua relação com a inovação nas organizações. Perspectivas em Ciência da Informação, 21(1), 97-118. 
Fialho, F. A. P., Macedo, M., Santos, N., \& Mitidieri, T. D. C. (2006). Gestão do conhecimento e aprendizagem: as estratégias competitivas da sociedade pós-industrial. Florianópolis: Visualbooks.

Fuks, H., Raposo, A. B., Gerosa, M. A., Pimentel, M., Filippo, D., \& Lucena, C. D. (2011). Teorias e modelos de colaboração. Sistemas colaborativos, 16-33.

Hinchcliffe, D. (2013) Today's enterprise collaboration landscape: Cloudy, social, mobile. Disponível em: <https://www.zdnet.com/article/todays-enterprise-collaborationlandscape-cloudy-social-mobile/>. Acesso em: 13 ago 2020.

Hinchcliffe, D. (2014). Defining the Next Generation Enterprise for 2014. Disponível em: $<$ https://dionhinchcliffe.com/2014/05/12/defining-the-next-generation-enterprise-for2014/>. Acesso em: 13 ago 2020.

Hinchcliffe, D. (2018). Four Strategic Frameworks for Digital Transformation. Disponível em: $<$ https://dionhinchcliffe.com/2018/10/18/four-strategic-frameworks-for-digitaltransformation/>. Acesso em: 13 ago 2020.

Intezari, A., Taskin, N., \& Pauleen, D. J. (2017). Looking beyond knowledge sharing: An integrative approach to knowledge management culture. J. Knowledge Management, 21(2), 492-515.

Mäntymäki, M., \& Riemer, K. (2016). Enterprise social networking: A knowledge management perspective. International Journal of Information Management, 36(6), 1042-1052.

McAfee, A. P. (2006). Enterprise 2.0: The dawn of emergent collaboration. Enterprise, 2, 1526.

Meira, S. R. De L. et al. (2011). Folksonomia. In: Sistemas Colaborativos. Rio de Janeiro: Elsevier.

Moffett, S., McAdam, R., \& Parkinson, S. (2004). Technological utilization for knowledge management. Knowledge and process management, 11(3), 175-184.

Motta, C. L. R., Garcia, A. C. B., Vivacqua, A. S., Santoro, F. M., \& Sampaio, J. O. (2011). Sistemas de recomendação. Pimentel, M.; Fuks, H. "Sistemas colaborativos". Rio de Janeiro: Elsevier.

Polizelli, D. L., \& Ozaki, A. M. (2008). Sociedade da informação: os desafios da era da colaboração e da gestão do conhecimento. São Paulo: Saraiva.

Probst, G., Raub, S., \& Romhardt, K. (2009). Gestão do conhecimento: os elementos construtivos do sucesso. Bookman Editora.

Rosini, A. M., \& Palmisano, A. (2013). Administração de sistemas de informação e a gestão do conhecimento. São Paulo. Cengage Learning; Edição: 2

Takeuchi, H., \& Nonaka, I. (2009). Gestão do conhecimento. Bookman Editora.

Von Krogh, G. (2012). How does social software change knowledge management? Toward a strategic research agenda. The Journal of Strategic Information Systems, 21(2), 154164.

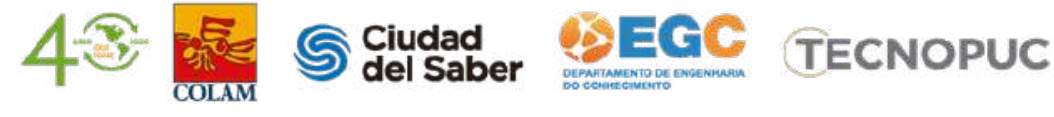


Wal, T. V. (2007) Folksonomy Coinage and Definition. Disponível em: $<$ http://www.vanderwal.net/folksonomy.html>. Acesso em: 30 mar. 2019.

Wal, T. V. (2018). 5 Core Insights for Community Platforms Today. Disponível em: $<$ https://www.personalinfocloud.com/blog/2018/9/27/core-insights-for-communityplatforms-today>. Acesso em: 22 maio. 2019.

Wenger, E., McDermott, R. A., \& Snyder, W. (2002). Cultivating communities of practice: A guide to managing knowledge. Harvard Business Press.

Wong, D., Bosua, R., Chang, S., \& Kurnia, S. (2016). Exploring the Use of Enterprise 2.0 and Its Impact on Social Capital within a Large Organisation.

Yazdi, M., \& Soltanali, H. (2019). Knowledge acquisition development in failure diagnosis analysis as an interactive approach. International Journal on Interactive Design and Manufacturing (IJIDeM), 13(1), 193-210.

Yin, R. K. (2015). Estudo de caso: Planejamento e métodos. Bookman editora. 